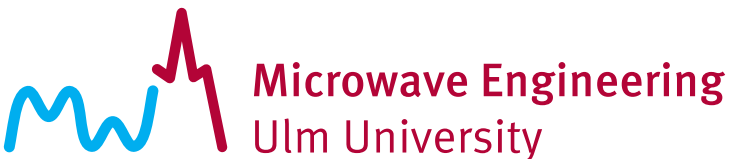

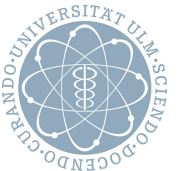

\section{Airborne Tripwire Detection using a Synthetic Aperture Radar}

\author{
Markus Schartel, Ralf Burr, Winfried Mayer, and Christian Waldschmidt
}

(C) 2019 IEEE. Personal use of this material is permitted. Permission from IEEE must be obtained for all other uses, in any current or future media, including reprinting/republishing this material for advertising or promotional purposes, creating new collective works, for resale or redistribution to servers or lists, or reuse of any copyrighted component of this work in other works. 


\title{
Airborne Tripwire Detection using a Synthetic Aperture Radar
}

\author{
Markus Schartel, Student Member, IEEE, Ralf Burr, Student Member, IEEE, \\ Winfried Mayer, Senior Member, IEEE, and Christian Waldschmidt, Senior Member, IEEE
}

\begin{abstract}
Anti-personnel fragmentation mines are relatively large metallic mines, which are only partially buried and often triggered by a metallic tripwire. In humanitarian mine clearance, the search for the wires is usually carried out manually. As a new approach, an airborne system for the detection of tripwires using a synthetic aperture radar is presented. The system consists of an industrial multicopter, a frequency-modulated continuouswave radar, and a real time kinematic global navigation satellite system. For image formation, a back-projection algorithm is used. Measurements with tripwires attached to a dummy mine successfully demonstrate the functionality of this system approach. In addition, the influence of wire length, vegetation, and incidence angle are investigated. It is shown that several overflights with different directions of flight are required to detect randomly oriented tripwires.
\end{abstract}

Index Terms-anti-personnel mine, frequency-modulated continuous-wave radar, multicopter, synthetic aperture radar, tripwire, unmanned aerial system.

\section{INTRODUCTION}

A NTI-PERSONNEL (AP) fragmentation mines pose a major lethal risk in humanitarian demining. In contrast to blast mines, fragmentation mines are much larger, have a high metallic content, and are usually either placed on the ground or only partially buried. They use an area fuzing system and have a large lethal range. A slight pull on a tripwire is enough to trigger the mine and injure or kill people in a radius of more than $50 \mathrm{~m}$ [1]. The mine itself should be very well detectable with metal detectors (MD), radars, or visually, but the challenge lies in the detection of hidden tripwires that trigger the mine from a distance.

The current mine clearance procedure for detecting and locating tripwires is carried out by hand with a tripwire feeler and by a visual search. The tripwire feeler is inserted parallel to the ground repeatedly and lifted slowly upwards. After searching for the tripwires, the vegetation is cut back. However, in dense vegetation this procedure has to be repeated multiple times. Moreover, this process is very time-consuming and requires maximum concentration, as one mistake could cost lives [2].

In addition to radars and metal detectors, chemical, acoustical, and optical sensors have been tested to detect landmines [3]. Likewise, various sensors have also been tested for

M. Schartel and C. Waldschmidt are with the Institute of Microwave Engineering, Ulm University, 89081 Ulm, Germany, e-mail: markus.schartel@uniulm.de.

R. Burr is with the Laboratory of Microtechnology, Ulm University of Applied Sciences, 89075 Ulm, Germany.

W. Mayer is with the Endress+Hauser SE+Co. KG, 79689 Maulburg, Germany the detection of tripwires. In [4], an acoustic method to detect non-metallic tripwires is proposed. The authors of [2] exploit the polarimetric infrared (IR) features of the light scattered and emitted in a scene to distinguish between man-made objects like tripwires and natural backgrounds. In [5], an imaging pulse radar mounted on a mobile platform is used to detect metal wires at stand-off range.

Due to the recent progress in the field of multicopters, it is now possible to integrate the above-mentioned sensors into an unmanned aerial system (UAS). The usage of an autonomous flying sensor platform in humanitarian demining enables the safe collection and analysis of data, before a deminer has to enter the hazardous environment.

In [6], partially buried landmines are detected optically using a down-looking on-board camera on a low-cost UAS. A UAS equipped with a metal detector and a thermographic camera is reported in [7]. UAS-based approaches using a downlooking ground penetrating radar (GPR) for mine detection are presented in [8], [9], [10]. The down-looking approach has the advantage that the radar can directly measure the depth of a target. However, the area throughput is low. The area under investigation has to be scanned in parallel straight lines (Bscans) so that a high-resolution $3 \mathrm{D}$ image (C-scan) can be generated. With regard to the detection of tripwires, depth resolution plays a subordinate role. Compared to the downlooking approach, the advantage of a side-looking radar is the increased area throughput and the lower ground reflections. In [11], different UASs designed for military applications in combination with a side-looking GPR are investigated for mine detection. However, due to the low altitude required to detect landmines with a GPR and the high material and financial risk associated with this type of UASs, the approach was considered impractical.

In this paper, a PROM-1 (Protupjeaka Rasprskavajua Odskona Mina-1) dummy mine is used as a test target. The PROM-1 is a bounding fragmentation AP mine manufactured in the Socialist Federal Republic of Yugoslavia [12], [13]. The housing of the mine consists of metal, weighs about $3 \mathrm{~kg}$, and has a total height of $27 \mathrm{~cm}$. A small primary explosion throws the mine in the air before it is shattered in all directions. The lethal range is more than $20 \mathrm{~m}$ in radius. Usually the mine is buried with the prongs $11 \mathrm{~cm}$ above ground. The PROM-1 can be triggered by up to 6 tripwires which are usually several meters in length with a diameter of less than $1 \mathrm{~mm}$. Generally, these tripwires are placed at a small distance parallel to the surface of the ground [5].

The focus of past research was either on the UAS-based 


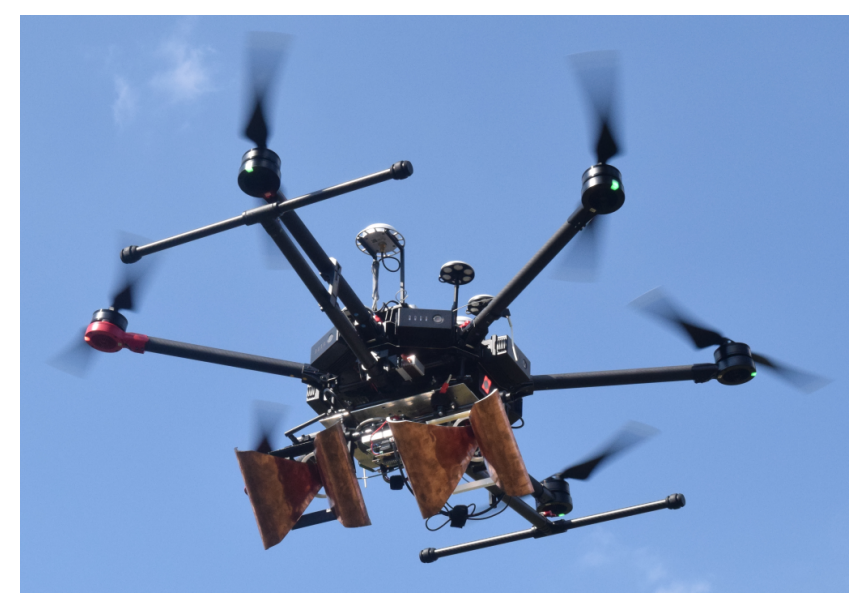

Fig. 1. UAS consisting of an industrial multicopter, an RTK GNSS, and an FMCW SAR.

detection of landmines or, in particular, on the detection of tripwires. In this work, the two topics are combined. In the following, a multicopter-based approach with a synthetic aperture radar (SAR) to detect PROM-1 fragmentation mines including their tripwires is investigated. First, the measurement system and the signal processing chain are briefly presented in Section II. In Section III, the measurement results with respect to wire length, incidence angle of the electromagnetic wave, and vegetation are discussed. Section IV gives a short conclusion.

\section{Measurement System}

\section{A. Hardware}

The measurement system employed is shown in Fig. 1. It consists of an industrial multicopter with an integrated inertial measurement unit (IMU) [14], a real time kinematic global navigation satellite system (RTK GNSS) with a horizontal and a vertical accuracy of $\pm 10 \mathrm{~mm}$ and $\pm 20 \mathrm{~mm}$, respectively [15], and a bistatic frequency-modulated continuouswave (FMCW) SAR operating in the frequency range from $1 \mathrm{GHz}$ to $4 \mathrm{GHz}$ [16]. The output data of the RTK GNSS $(5 \mathrm{~Hz})$, the multicopter integrated IMU $(50 \mathrm{~Hz})$, and the SAR $(30 \mathrm{~Hz})$ are time-stamped and stored on a single-board computer for offline processing.

The transmit power of the radar is $P_{\mathrm{T}}=15 \mathrm{dBm}$ and the ramp time of the linear frequency chirp is $T=1 \mathrm{~ms}$. The beat signal is sampled with a $4 \mathrm{MHz}$ analog-to-digital converter. The maximum chirp repetition frequency (CRF) is currently limited to $30 \mathrm{~Hz}$ by the data transfer between the radar and the single-board computer used for data storage.

The lightweight 3D-printed horn antennas can be manually rotated by $360^{\circ}$ to change the polarization between the flights in a few seconds [17]. The frequency-dependent $3 \mathrm{~dB}$ beamwidth is approximately $60^{\circ}$ in the E-plane and $50^{\circ}$ in the $\mathrm{H}$-plane and the gain of the antenna is approximately $6 \mathrm{dBi}$.

The flight time of the multicopter with the payload of $2.3 \mathrm{~kg}$ is about $23 \mathrm{~min}$. Currently, most flights are operated manually, with the exception of simple patterns, which can be flown automatically using a mobile application from the

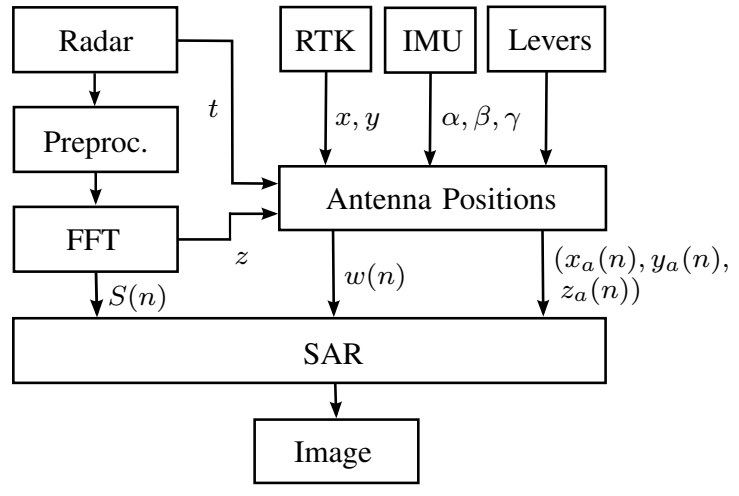

Fig. 2. Signal processing tool chain.

manufacturer. The first multicopter-based SAR and GPSAR measurement results are presented in [18], [19].

\section{B. Signal Processing}

Due to the highly non-linear flight path of the multicopter, a standard back-projection algorithm is used for the image formation [20]. The image at the location $\left(x_{0}, y_{0}, z_{0}\right)$ results from

$$
\operatorname{im}\left(x_{0}, y_{0}, z_{0}\right)=\sum_{n=1}^{N} w(n) S(n) \exp \left(-\mathrm{j} \phi_{x_{0}, y_{0}, z_{0}}\right),
$$

where $N$ is the number of chirps, $w$ a weighting factor, $S$ the interpolated and range-compressed radar signal, and $\phi$ the expected phase for the pixel. The expected phase is given by

$$
\phi_{x_{0}, y_{0}, z_{0}}=2 \pi f_{0} \Delta t-\pi K \Delta t,
$$

with $f_{0}$ being the start frequency, $K$ the slope of the chirp, and $\Delta t$ the round-trip time. $\Delta t$ results from the distance between the antenna and the target

$$
\Delta t=\frac{2}{c_{0}} \sqrt{\left(x_{a}-x_{0}\right)^{2}+\left(y_{a}-y_{0}\right)^{2}+\left(z_{a}-z_{0}\right)^{2}},
$$

with the speed of light $c_{0}$ and the position of the antenna during a chirp $\left(x_{a}, y_{a}, z_{a}\right)$. For each chirp, the algorithm requires the exact antenna position with respect to the the target pixel $\left(x_{0}, y_{0}, z_{0}\right)$, where $x_{0}$ and $y_{0}$ are the pixel coordinates in the 2D-SAR image. In the case of a flat surface, $z_{0}$ is zero.

In the following, the signal processing chain depicted in Fig. 2 is described in more detail. Depending on the desired frequency range, the time domain radar data are truncated. After the range offset is corrected by multiplying the time domain signal with a complex correction term, the data are range-compressed and interpolated by performing a Hannwindowed fast Fourier transform with 16-fold zero-padding. The result of this pre-processing chain is $S(n)$.

The next step is to estimate the antenna positions $\left(x_{a}(n), y_{a}(n), z_{a}(n)\right)$ for each chirp $n$. The altitude $z$ is extracted from the range-compressed radar data $S(n)$. The $x$ and $y$ components are obtained from the RTK GNSS. Then, $x, y, z$, the lever arms from the IMU to the antennas, and the Euler angles $\alpha, \beta, \gamma$ provided by the IMU are used to calculate the position of the phase centers $\left(x_{a}, y_{a}, z_{a}\right)$ via translations and 
rotations. Afterwards, the position data are interpolated to fit to the timestamps of the radar data.

The weighting factor $w(n)$ of each chirp is chosen proportionally to the distance to the previous chirp. The longer the distance, the larger the weighting factor. This ensures that the radar image is not distorted by changing flight speeds.

\section{Measurement Results}

A slow airspeed (or a high CRF) increases the available integration time and ultimately improves the sensor sensitivity. The detection performance is then limited to the signal-toclutter ratio only. A prerequisite for coherent SAR integration, however, is very accurate positioning throughout the integration time. In these measurements, the multicopter was controlled with the aim of achieving a trajectory as straight as possible (linear SAR) at a constant speed of about $2 \mathrm{~m} / \mathrm{s}$ in order to comply with the sampling theorem and to achieve maximum efficiency in terms of area per time.

For the evaluation of the following measurement results, the operating frequency of the radar was truncated from $1 \mathrm{GHz}$ to $2.3 \mathrm{GHz}$ due to the frequency-dependent behaviour of the signature of the tripwires. The antennas were mounted in horizontal polarization. The diameter of the tripwires was $0.5 \mathrm{~mm}$.

\section{A. Angle and Length Dependencies}

The radar cross-section (RCS) of tripwires is reduced in the case of oblique incidence [5]. However, in real applications, the orientation of the tripwires is arbitrary and unknown, and depending on the angle of incidence, the wire may not be visible at all. In order to ensure that all tripwires are detected, the area to be investigated must be overflown several times with different flight directions. To analyze the angular system performance, tripwires with a length of $3 \mathrm{~m}$ were tensioned at an angle of $0^{\circ}, 5^{\circ}, 20^{\circ}, 25^{\circ}$, and $40^{\circ}$ at a height of approximately $15 \mathrm{~cm}$ parallel to the ground. The measurement setup and the measured trajectory projected on the ground plane are shown in Fig. 3. The wires were attached to small

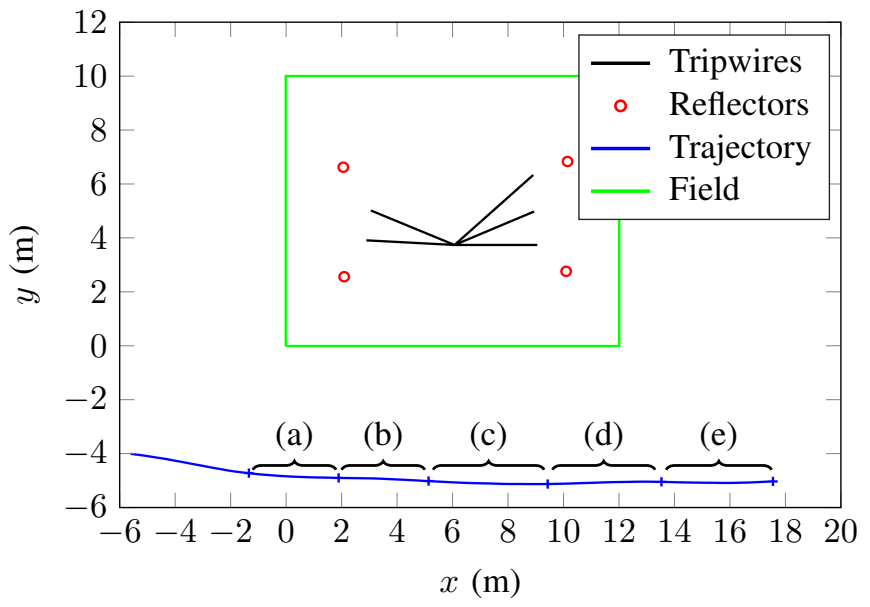

Fig. 3. Sketch of the measurement setup to analyze the angular system performance. (a)-(e) show the sub-apertures.

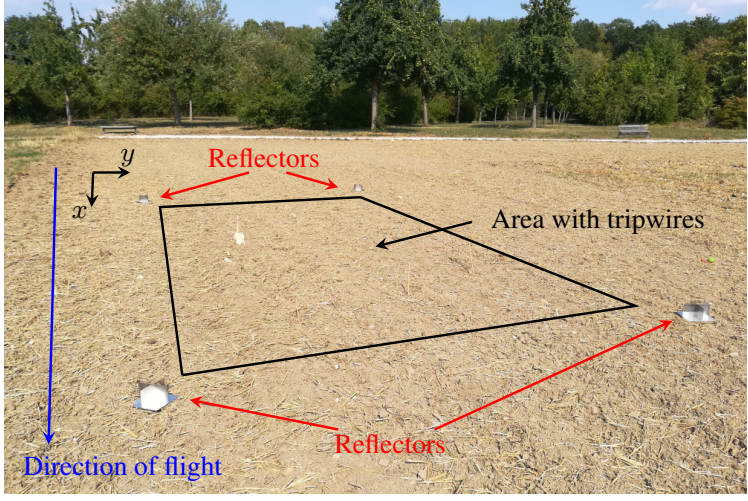

Fig. 4. Photo of the measurement setup sketched in Fig. 3.

wooden rods over a freshly ploughed field without vegetation. A photo of the setup is shown in Fig. 4.

The UAS was flown manually parallel to the measurement setup. In total 351 chirps were recorded leading to a total length of the synthetic aperture of $L_{\mathrm{SAR}}=23 \mathrm{~m}$. Regarding the minimum wavelength $\lambda_{\min }=0.13 \mathrm{~m}$ and the mean sampling distance $\overline{\Delta x}=0.065 \mathrm{~m}$, the sampling theorem is fulfilled. The range compressed radar data (range profile) and the extracted altitude of the UAV are shown in Fig. 5. The range profiles are plotted versus the chirp number, where the first chirp was recorded at position $(x, y)=(-5.5 \mathrm{~m},-4 \mathrm{~m})$ and the last chirp was recorded at the position $(17.5 \mathrm{~m},-5 \mathrm{~m})$ as illustrated in Fig. 3.

The strong crosstalk of the antennas can be recognized in the range up to about $R=3 \mathrm{~m}$. Therefore, the gain of the receiver chain is limited to avoid clipping. The continuous reflection at about $3 \mathrm{~m}$ corresponds to the altitude of the UAV. In the range of about $5 \mathrm{~m}$ to $7 \mathrm{~m}$, the border between meadow and field is visible.

The reflectors are targets that are visible over a wide angular range, along almost the full length of the synthetic aperture. Their reflections lead to the four hyperbolas cleary visible in the image. In contrast, the wires are extended targets. They

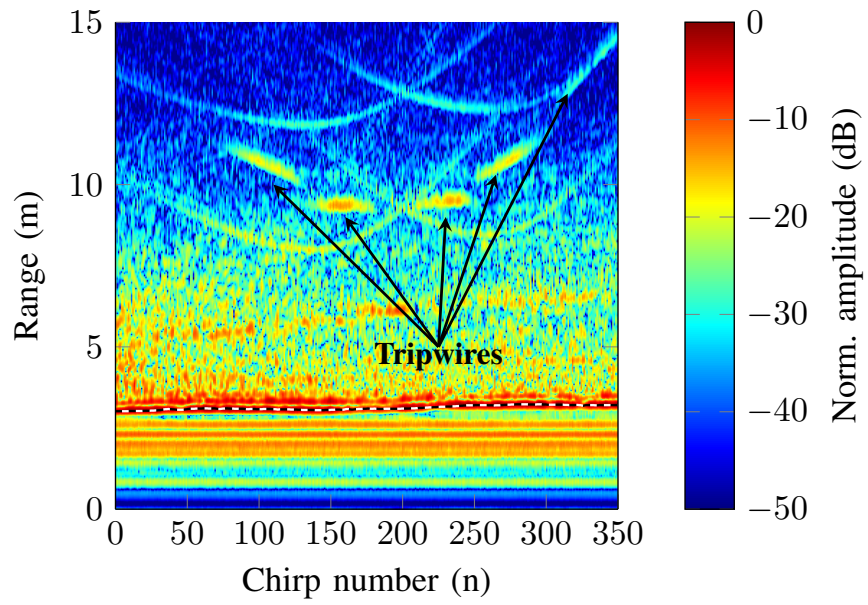

Fig. 5. Range compressed radar data and the extracted altitude of the UAV (----). 

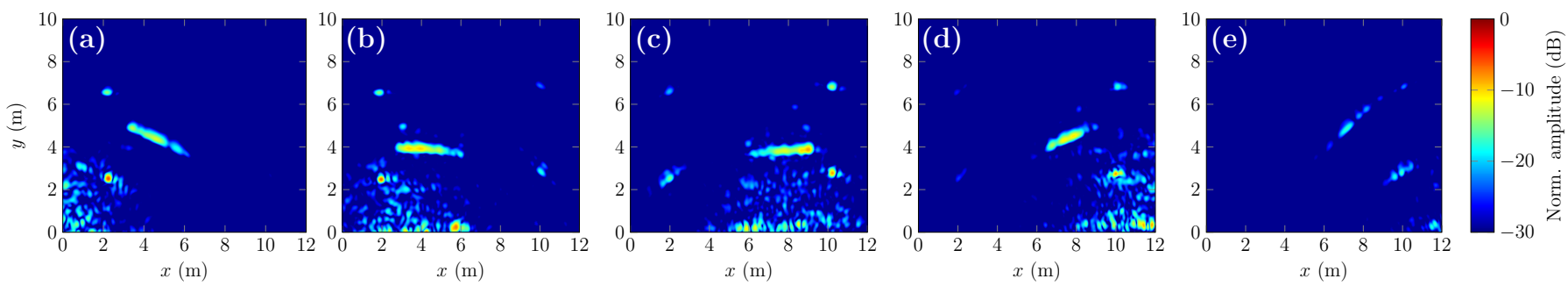

Fig. 6. Processed sub-apertures of the data shown in Fig. 5. (a) from chirp 78 to 132, (b) 132 to 183 , (c) 183 to 245 , (d) 245 to 300 , and (e) 300 to 350 .

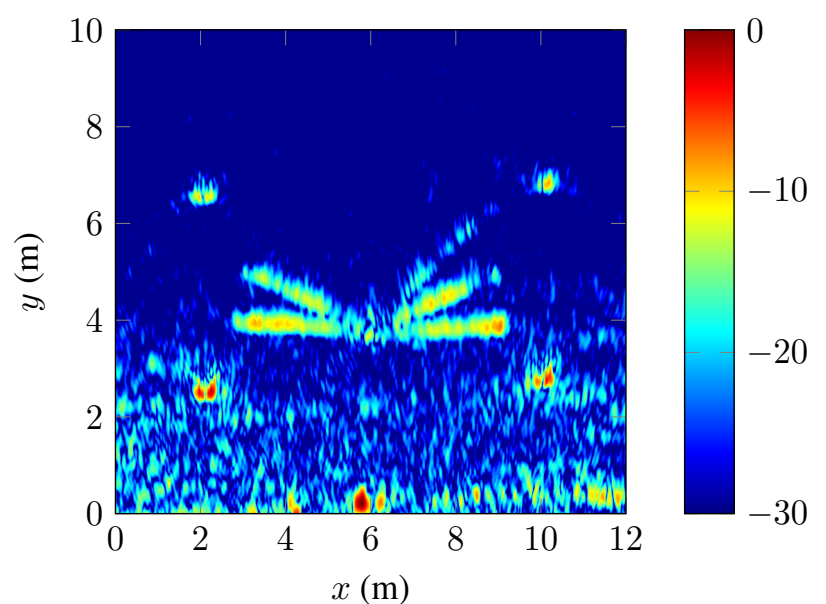

Fig. 7. SAR image of the data shown in Fig. 5 processed along the full length of the synthetic aperture.

are only visible in a few chirps, as they are only detectable from a small angular range.

Their RCS strongly depends on the angle of incidence. To further investigate this effect, the radar data are divided into sub-apertures shown in Fig. 6.

Although the 2 wires on the left side (Fig. 6 (a) and (b)) and the 3 on the right side (Fig. 6 (c), (d) and (e)) are in the same $x$-range, each of them can only be detected if the angle of incidence is close to normal.

The signature of the tripwire in Fig. 6 (e) is limited due to the increasing distance between radar and target, the finite length of the aperture, and the radiation pattern of the antenna. This measurement clearly shows the limits of the UAS.

The SAR image processed along the full length of the synthetic aperture is shown in Fig. 7. For reliable detection of randomly oriented tripwires, 4 overflights with a flight direction of $0^{\circ}, 45^{\circ}, 90^{\circ}$, and $135^{\circ}$ are recommended. The best result can be achieved with circular SAR, as the wire is illuminated from all possible angles.

In addition to the orientation, the tripwire length influences the RCS value. To investigate this effect, 4 tripwires with lengths of $25 \mathrm{~cm}, 50 \mathrm{~cm}, 1 \mathrm{~m}$, and $2 \mathrm{~m}$ have been tensioned at a height of approximately $15 \mathrm{~cm}$ parallel to the ground and parallel to the flight direction. The experimental setup was carried out on the same site as shown in Fig. 4 and the resulting SAR image is shown in Fig. 8.

The tripwires of a PROM-1 are a few meters long [21]. The measurement result shows that all but the shortest wire with a

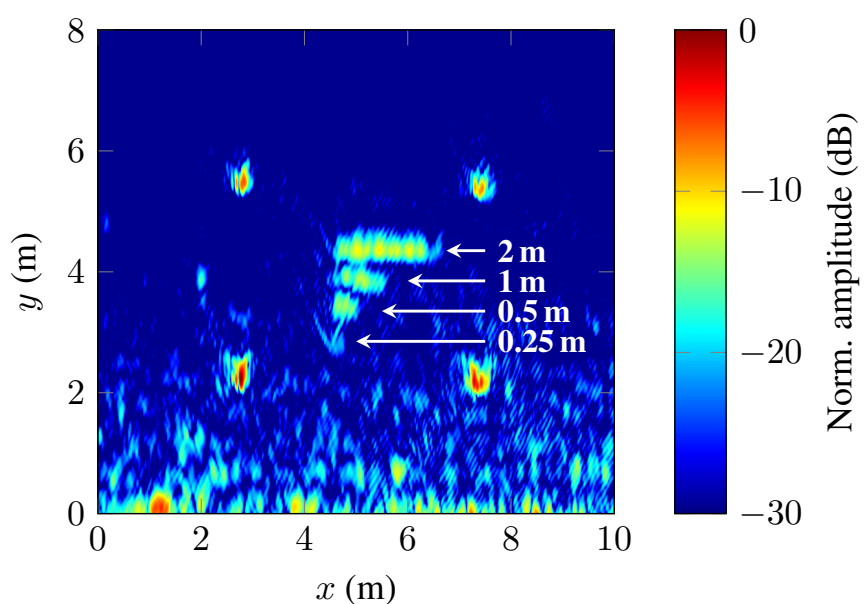

Fig. 8. SAR image of a measurement setup to investigate the influence of the wire length.

length of $25 \mathrm{~cm}$ are clearly visible. Thus, with respect to the RCS, the length of the wire is not of critical concern. With regard to a classification, it is obvious that from a length of $1 \mathrm{~m}$ and more, the shape of a wire could be very well deduced.

\section{B. Influence of Vegetation}

In order to simulate a more realistic scenario, a metal bottle was used as a PROM-1 dummy. The prongs and fuze extension were made of brass and aluminium, respectively. The total height of the dummy is about $33 \mathrm{~cm}$. It was buried about halfway and 4 wires with a length of $3 \mathrm{~m}$ were tensioned at a height of about $15 \mathrm{~cm}$ to $18 \mathrm{~cm}$ above ground. Four corner reflectors were placed as reference. The measurements were made early in the morning, so the grass was still very wet with dew. A picture of the setup is shown in Fig. 9. Although the wires have a red sheath, they are hardly visible.

Several overflights in different altitudes and with different directions of flight have been performed. Depending on the flight direction, at least 2 , or in the best case all 4 wires were recognized. In comparison to the previous unvegetated measurements (cf. Fig. 7), the reflected power of the wires is significantly reduced by the vegetation as can be seen in Fig. 10.

Thus, a reliable detection cannot be guaranteed. To overcome this problem, single-look radar images are combined using an incoherent addition to create a multi-look image. In advance, the distance-dependent signal attenuation is compensated. For comparison, a multi-look image consisting of 4 


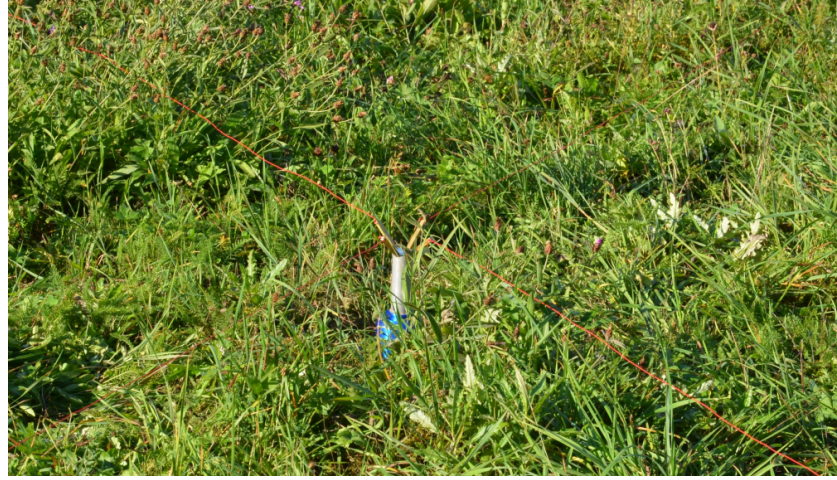

Fig. 9. Measurement setup consisting of a PROM-1 dummy and 4 tripwires in wet grass, covered with dew.

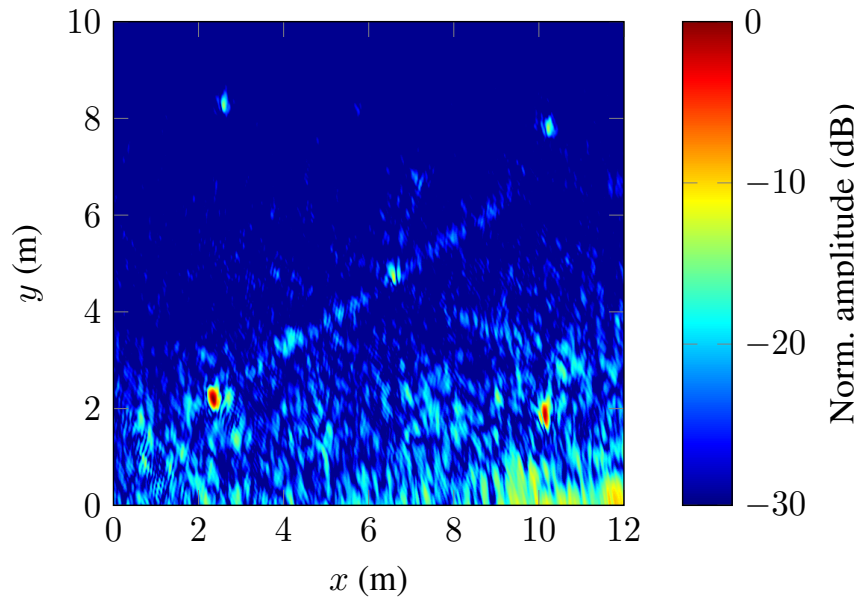

Fig. 10. Single-look stripmap SAR image of the setup shown in Fig. 9.

single-looks is shown in Fig. 11. The PROM-1 dummy in the center of the 4 reflectors as well as all 4 wires are reliably detectable.

\section{CONCLUSION}

In this work, a functional multicopter-based SAR system for the detection of metallic tripwires was presented for the first time. It was shown that randomly orientated, vegetationobscured tripwires can be detected by overflying the area in four different directions. Both the wire length and the vegetation have a strong influence on the signal strength. However, the multi-look processing ensures reliable detection. In areas with light vegetation, this system could detect tripwires before a deminer has to enter the potentially hazardous area and thus accelerate the process of mine clearance. Currently, an area throughput of approximately $10 \mathrm{~m}^{2} / \mathrm{s}$ is achieved (single-look). In order to be able to operate the system in a real minefield, the focus is on automatic or ideally autonomous flight operations.

\section{REFERENCES}

[1] C. King, "Demining: enhancing the process," in Second International Conference on the Detection of Abandoned Land Mines, Oct. 1998

[2] J. Storm and T. Geisler, "Trip wire detection using polarimetric IR," in Proc. SPIE 4038, Detection and Remediation Technologies for Mines and Minelike Targets V, 2000.

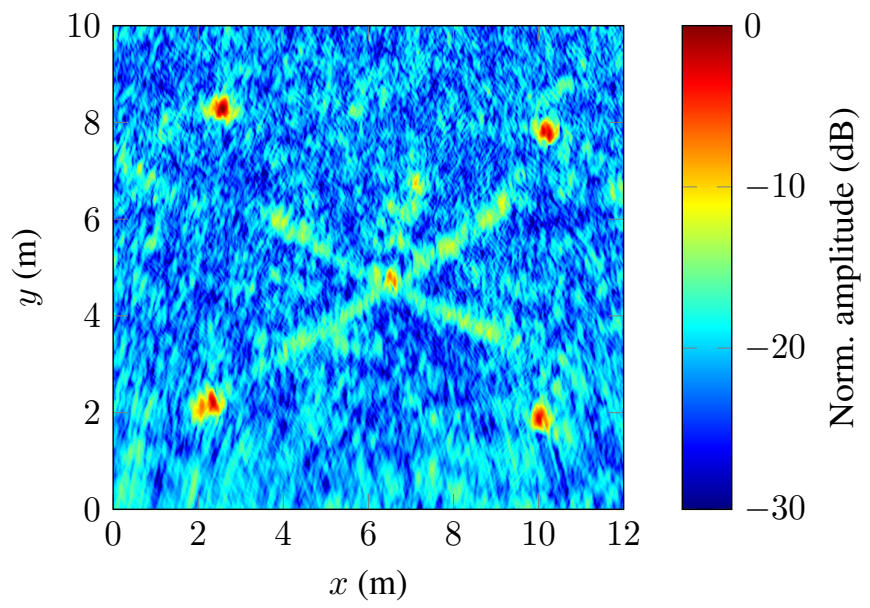

Fig. 11. Multi-look SAR image of the setup shown in Fig. 9. 4 SAR images were added incoherently.

[3] L. Robledo, M. Carrasco, and D. Mery, "A survey of land mine detection technology," Int. J. Remote Sens., vol. 30, no. 9, pp. 2399-2410, 2009.

[4] L. J. Carter and C. Y. Liao, "A new approach to detecting vegetationobscured tripwires," in First Int. Conf. on Industrial and Information Systems, Aug. 2006, pp. 619-624.

[5] G. N. Crisp, C. R. Thornhill, and G. Gooding-Williams, "Detection of Wires Using UWB Radar,' in Ultra-Wideband, Short-Pulse Electromagnetics 6. Boston, MA: Springer US, 2003, pp. 471-480.

[6] C. Castiblanco et al., "Air Drones for Explosive Landmines Detection," in ROBOT2013: First Iberian Robotics Conference, 2014, pp. 107-114.

[7] Y. Ganesh, R. Raju, and R. Hegde, "Surveillance Drone for Landmine Detection," in Int. Conf. on Adv. Computing and Commun., Sep. 2015, pp. 33-38.

[8] J. Colorado et al., "An integrated aerial system for landmine detection: SDR-based Ground Penetrating Radar onboard an autonomous drone," Adv. Rob., vol. 31, no. 15, pp. 791-808, 2017.

[9] G. Fasano et al., "Proof of concept of micro-UAV-based radar imaging," in Int. Conf. on Unmanned Aircraft Systems, Jun. 2017, pp. 1316-1323.

[10] M. G. Fernández et al., "Synthetic Aperture Radar Imaging System for Landmine Detection Using a Ground Penetrating Radar on Board a Unmanned Aerial Vehicle," IEEE Access, vol. 6, pp. 45 100-45 112, 2018.

[11] A. Goad et al., "Landmine detection utilizing an unmanned aerial vehicle," in IEEE Systems and Information Engineering Design Symposium, Apr. 2008, pp. 231-236.

[12] The Silent Menace: Landmines in Bosnia and Herzegovina. International Committee of the Red Cross (ICRC), 1998.

[13] M. Fernandez, A. Lewis, and F. Littmann, "Prom 1 anti-personnel landmines; possibility of activation by physical contact with a metal detector," Joint Research Centre - European Commission (JRC/EC), Tech. Rep., 2001.

[14] DJI, "Matrice 600 Pro User Manual v1.0," 2017.

[15] DJI, "D-RTK Release Notes," 2017.

[16] R. Burr et al., "Design and Implementation of a FMCW GPR for UAVbased Mine Detection," in IEEE MTT-S International Conference on Microwaves for Intelligent Mobility, 2018.

[17] R. Burr et al., "Lightweight Broadband Antennas for UAV based GPR Sensors," in European Microwave Conference, Oct. 2018.

[18] M. Schartel et al., "UAV-Based Ground Penetrating Synthetic Aperture Radar," in IEEE MTT-S International Conference on Microwaves for Intelligent Mobility, 2018.

[19] M. Schartel et al., "A Multicopter-Based Focusing Method for Ground Penetrating Synthetic Aperture Radars," in IEEE International Geoscience and Remote Sensing Symposium, Jul. 2018.

[20] E. C. Zaugg and D. G. Long, "Generalized Frequency Scaling and Backprojection for LFM-CW SAR Processing," IEEE Trans. Geosci. Remote Sens., vol. 53, no. 7, pp. 3600-3614, Jul. 2015.

[21] C. King, Jane's Mines \& EOD Operational Guide. IHS Jane's, 2018. 